\title{
Pstat: An R Package to Assess Population Differentiation in Phenotypic Traits
}

by Stéphane Blondeau Da Silva and Anne Da Silva

\begin{abstract}
The package Pstat calculates $P_{S T}$ values to assess differentiation among populations from a set of quantitative traits and provides bootstrapped distributions and confidence intervals for $P_{S T}$. Variations of $P_{S T}$ as a function of the parameter $c / h^{2}$ are studied as well. The package implements different transformations of the measured phenotypic traits to eliminate variation resulting from allometric growth, including calculation of residuals from linear regression, Reist standardization, and the Aitchison transformation.
\end{abstract}

\section{Introduction}

Understanding the causes governing patterns of morphological variations in the wild represents a fundamental goal of evolutionary biology. In particular, the relative importance of selective and neutral processes behind the observed differentiation remains a crucial question.

Studies comparing differentiation in quantitative traits and neutral markers have significantly increased over the last ten years (Leinonen et al., 2013). Typically, a set of populations is sampled and the degree of genetic differentiation is estimated for a set of molecular markers with the Wright's $F_{S T}$ index (Wright, 1951). For its part, the $Q_{S T}$ index (Spitze, 1993) assesses the degree of phenotypic differentiation over a set of quantitative traits. The logic of $F_{S T}$ and $Q_{S T}$ comparison relies on the assumption that the $F_{S T}$ obtained by the consideration of neutral markers reflects the divergence only induced by genetic drift (Reynolds et al., 1983). Hence, $F_{S T}$ provides a null expectation and allows estimation of the degree of population differentiation that would be reached without selection (Merilä and Crnokrak, 2001).

As a consequence, the comparison between $F_{S T}$ and $Q_{S T}$ leads to three possibilities: (i) $Q_{S T}>F_{S T}$ means that quantitative traits show a higher level of differentiation than what would have been expected under the influence of genetic drift, such that natural selection could induce differentiation between populations by favoring different phenotypes (i.e., heterogeneous selection); (ii) $Q_{S T}<F_{S T}$ could indicate the influence of natural selection, but selecting for same optima among populations (i.e., homogeneous selection); (iii) $Q_{S T}=F_{S T}$ means that no departure from neutral expectations can be detected and that the degree of differentiation in quantitative traits could have been obtained only by genetic drift, even if the contribution of natural selection can neither be excluded nor estimated.

Spitze (1993) introduced and defined the $Q_{S T}$ quantity as follows for diploid species assuming purely additive gene action:

$$
Q_{S T}=\frac{\sigma_{A_{b}}^{2}}{\sigma_{A_{b}}^{2}+2 \sigma_{A_{w}}^{2}},
$$

where $\sigma_{A_{b}}^{2}$ and $\sigma_{A_{w}}^{2}$ are the morphological additive genetic variance components between and within populations.

In the wild, the estimation of the additive genetic variance components is challenging as breeding design is impossible. Therefore, $Q_{S T}$ is often approximated by $P_{S T}$ (Leinonen et al., 2006), which is directly calculated from the total phenotypic variance components with no distinction between the relative contribution of genetic and environmental variations:

$$
P_{S T}=\frac{c \sigma_{b}^{2}}{c \sigma_{b}^{2}+2 h^{2} \sigma_{w}^{2}} \quad \text { or } \quad P_{S T}=\frac{\frac{c}{h^{2}} \sigma_{b}^{2}}{\frac{c}{h^{2}} \sigma_{b}^{2}+2 \sigma_{w}^{2}},
$$

where $\sigma_{b}^{2}$ and $\sigma_{w}^{2}$ are the respective phenotypic variances between and within populations, $c$ is an estimate of the proportion of the total variance due to additive genetic effects across populations, and $h^{2}$ is heritability, the proportion of phenotypic variance due to additive genetic effects (Brommer, 2011). How well $P_{S T}$ approximates $Q_{S T}$ depends on the parameters $c$ and $h^{2}$, such that if the values of $c$ and $h^{2}$ are known, then the phenotypic divergence quantified by $P_{S T}$ would equal $Q_{S T}$. This implies caution in the interpretation obtained from $P_{S T}$ (Brommer, 2011).

A large number of studies have assessed the potential for natural selection to affect morphological evolution by comparing phenotypic divergence with neutral genetic divergence via a $P_{S T}$ versus $F_{S T}$ approach (e.g., Mobley et al., 2011; Lima, 2012; He et al., 2013; Shinn et al., 2015). While estimation of $F_{S T}$ values are included in various R packages such as diveRsity (Keenan et al., 2013) or hierfstat 
(Goudet, 2005), no R package exists to deal with the $P_{S T}$ index. In this study, we present the Pstat package to handle large datasets of quantitative traits and correct quantitative traits taking into account allometric growth. The package calculates $P_{S T}$ values with their respective bootstrapped confidence intervals, and offers several options to select individuals, traits, or populations. We also provide various plotting tools for the visual evaluation of $P_{S T}$ and $F_{S T}$ values. We will walk through a detailed example to give an overview of the Pstat package.

\section{An example to get familiar with the main functions}

After loading the package with library (Pstat), load the sample data with data(test). This data frame contains 200 rows, with each row representing an individual in a population of common wetland plants, Juncus effusus (see Michalski and Durka 2015 and the Dryad Digital Repository, https://doi.org/10.5061/dryad. bk5hk). The data frame contains the name of the populations (A, B, $C, D$, and E) to which each individual belongs and eleven quantitative measures. An excerpt from the sample data are presented in Table 1.

\begin{tabular}{ccccccc}
\hline & Populations & QM1 & QM2 & QM3 & QM4 & $\ldots$ \\
\hline 1 & A & 0.18487253 & 0.4001979 & 0.1694021 & 42 & $\ldots$ \\
2 & B & 0.24023500 & 0.4718000 & 0.2178500 & 46 & $\ldots$ \\
3 & C & 0.23499676 & 0.4686213 & 0.2060222 & 25 & $\ldots$ \\
4 & B & 0.20495223 & 0.3746026 & 0.1846816 & 51 & $\ldots$ \\
5 & C & 0.20739220 & 0.4866461 & 0.2131618 & 19 & $\ldots$ \\
6 & C & 0.22545341 & 0.3770903 & 0.1882165 & 28 & $\ldots$ \\
7 & C & 0.18371681 & 0.4992361 & 0.2167194 & 25 & $\ldots$ \\
$\ldots$ & $\ldots$ & $\ldots$ & $\ldots$ & $\ldots$ & $\ldots$ & $\ldots$ \\
\hline
\end{tabular}

Table 1: Sample from the test data frame, containing quantitative measures for individual members of Juncus effusus (Michalski and Durka, 2015).

\section{The data preparation}

The package can be used to transform data to eliminate variation resulting from allometric growth. Users have the choice between three alternatives:

1. Residuals of a linear regression, with one of the quantitative variables used as the regressor (Kuhry and Marcus, 1977);

2. The allometric transformation described in Reist (1985); or

3. Aitchison's log-ratio transform (Aitchison, 1986).

Among a variety of univariate transformations that aim to separate size and shape variations, Reist (1985) showed that adjustments for size using a regression and residuals (the first option) and allometric adjustments to a standard size (the second option) are preferred since they allow the complete removal of size variations and have minimal impact on the correlation and covariance structure of the data. Unlike the first two options, the third transformation offers the benefit of keeping the same number of variables. We provide examples of each of the three alternatives below.

\section{Simple linear adjustments}

The first adjustment method provided by Pstat is a simple linear regression. Assuming the existence of linear relationships between the dependent variable and one of the quantitative traits, the Res function returns a new data frame with the residuals of the regression. The function's arguments are as follows:

- data: the studied data frame to be transformed with as many rows as individuals; the first column must contain the population to which the individual belongs and the other columns may contain quantitative variables.

○ reg: the name or the rank of the variable chosen as the regressor.

- Rp: the names of the populations to be deleted. Default value: $\mathrm{Rp}=0$, no population removed.

- Ri: the line numbers of individuals to be deleted. Default value: $\mathrm{Ri}=0$, no individuals removed. 
We present sample output from the test data, using one of the quantitative traits as the regressor. A sample of the transformed data output by Res is presented in Table 2.

\#\# Using the explanatory variable QM3 as the regressor Res (data=test, reg="QM3")

\begin{tabular}{cccccc}
\hline & Populations & QM1 & QM2 & QM4 & $\ldots$ \\
\hline 1 & A & 0.0339245264 & $5.621424 \mathrm{e}-03$ & 6.23817063 & $\ldots$ \\
2 & $\mathrm{~B}$ & 0.1001268662 & $4.497085 \mathrm{e}-02$ & 8.44522196 & $\ldots$ \\
3 & $\mathrm{C}$ & 0.0922422473 & $4.966613 \mathrm{e}-02$ & -12.11705813 & $\ldots$ \\
4 & $\mathrm{~B}$ & 0.0574228940 & $-3.014565 \mathrm{e}-02$ & 14.67271191 & $\ldots$ \\
5 & $\mathrm{C}$ & 0.0662351079 & $6.293798 \mathrm{e}-02$ & -18.38127681 & $\ldots$ \\
6 & $\mathrm{C}$ & 0.0787149904 & $-3.001126 \mathrm{e}-02$ & -8.45810788 & $\ldots$ \\
7 & $\mathrm{C}$ & 0.0433557311 & $7.315960 \mathrm{e}-02$ & -12.51293868 & $\ldots$ \\
$\ldots$ & $\ldots$ & $\ldots$ & $\ldots$ & $\ldots$ & $\ldots$ \\
\hline
\end{tabular}

Table 2: Sample from the adjusted data frame output by Res, using QM3 as the explanatory variable.

\section{Reist transformation}

In the second adjustment method provided by Pstat, all morphometric measurements are standardized using the transformation proposed by Reist (1985).

Let $n$ be the number of individuals and $p$ the number of quantitative traits such that $\exists k \in$ $\{1, \ldots, p\}$ and the $k^{\text {th }}$ trait is the explanatory variable. Let us denote this variable $\left(x_{i}\right)_{1 \leq i \leq n}$ and the other traits as $j \in\{1, \ldots, p\} \backslash\{k\},\left(y_{i j}\right)_{1 \leq i \leq n}$. The Reist transformation is

$$
\begin{aligned}
& \forall i \in\{1, \ldots, n\} \text { and } \forall j \in\{1, \ldots, p\} \backslash\{k\}, \\
& Y_{i j}=\log \left(y_{i j}\right)-b_{j}\left(\log \left(x_{i}\right)-\log (\bar{x})\right),
\end{aligned}
$$

where $Y_{i j}$ is the size adjusted measurement of the $j^{\text {th }}$ trait for the $i^{\text {th }}$ individual, $y_{i j}$ the original morphometric measurement, $\bar{x}$ the population mean of the explanatory variable, and $x_{i}$ the value of the explanatory variable for the $i^{\text {th }}$ individual. For all $j \in\{1, \ldots, p\} \backslash\{k\}$, the parameter $b_{j}$ is estimated for the quantitative trait $y_{j}$ (i.e. $\left(y_{i j}\right)_{1 \leq i \leq n}$ ) and represents the slope of the linear regression of $\log \left(y_{j}\right)$ on $\log (x)$.

The ReistTrans function returns a corrected data frame. Using QM3 as the explanatory variable, we present a sample of the transformed data frame in Table 3.

\#\# Using QM3 as the explanatory variable (identified by column number)

ReistTrans(test, reg=3)

\begin{tabular}{cccccc}
\hline & Populations & QM1 & QM2 & QM4 & $\ldots$ \\
\hline 1 & A & -0.7445410 & -0.3859875 & 1.631722 & $\ldots$ \\
2 & B & -0.6004703 & -0.3462059 & 1.648348 & $\ldots$ \\
3 & C & -0.6167708 & -0.3421063 & 1.388608 & $\ldots$ \\
4 & B & -0.6893556 & -0.4255755 & 1.708186 & $\ldots$ \\
5 & C & -0.6669355 & -0.3300087 & 1.266323 & $\ldots$ \\
6 & C & -0.6456670 & -0.4250906 & 1.446049 & $\ldots$ \\
7 & C & -0.7175846 & -0.3210021 & 1.384003 & $\ldots$ \\
$\ldots$ & $\ldots$ & $\ldots$ & $\ldots$ & $\ldots$ & $\ldots$ \\
\hline
\end{tabular}

Table 3: Sample from the Reist adjusted data frame using QM3 as the explanatory variable.

\section{Aitchison transformation}

The third adjustment method provided by Pstat performs the Aitchison log-ratio transformation to account for individual size-effects (Aitchison, 1986).

Let $n$ be the number of individuals and $p$ the number of morphological traits. For $j \in\{1, \ldots, p\}$, let $\left(y_{i j}\right)_{1 \leq i \leq n}$ represent the quantitative variables. The formula formula for the Aitchison transformation 
is as follows:

$$
\begin{aligned}
& \forall i \in\{1, \ldots, n\} \text { and } \forall j \in\{1, \ldots, p\}, \\
& Y_{i j}=\log \left(y_{i j}\right)-\frac{1}{p} \sum_{k=1}^{p} \log \left(y_{i k}\right),
\end{aligned}
$$

where $Y_{i j}$ is the transformed measure of the $j^{\text {th }}$ trait for the $i^{\text {th }}$ individual, and $y_{i j}$ is the original value for the $i^{\text {th }}$ individual and the $j^{\text {th }}$ trait.

The AitTrans function returns a corrected data frame. Sample output are included in Table 4.

AitTrans(test)

\begin{tabular}{ccccccc}
\hline & Populations & QM1 & QM2 & QM3 & QM4 & $\ldots$ \\
\hline 1 & A & -1.947544 & -1.6121417 & -1.985498 & 0.408832854 & $\ldots$ \\
2 & B & -1.910214 & -1.6170919 & -1.952692 & 0.371908012 & $\ldots$ \\
3 & C & -1.834151 & -1.5343910 & -1.891299 & 0.192727037 & $\ldots$ \\
4 & $\mathrm{~B}$ & -1.901481 & -1.6395625 & -1.946710 & 0.494436831 & $\ldots$ \\
5 & $\mathrm{C}$ & -1.832709 & -1.4622885 & -1.820792 & 0.129251912 & $\ldots$ \\
6 & $\mathrm{C}$ & -1.801889 & -1.5785008 & -1.880288 & 0.292211929 & $\ldots$ \\
7 & $\mathrm{C}$ & -1.938699 & -1.5045418 & -1.866950 & 0.195092172 & $\ldots$ \\
$\ldots$ & $\ldots$ & $\ldots$ & $\ldots$ & $\ldots$ & $\ldots$ & $\ldots$ \\
\hline
\end{tabular}

Table 4: Sample from the Aitchison adjusted data frame.

\section{Phenotypic differentiation evaluation and confidence intervals}

\section{$P_{S T}$ values}

We are interested in determining the phenotypic differentiation across the five populations for each of the eleven quantitative traits of the example dataset. The function Pst can determine the $P_{S T}$ values of each trait with the associated bootstrapped confidence intervals (Efron and Tibshirani, 1993). The arguments to Pst are as follows:

o data: the input data frame with as many rows as individuals; the first column must contain the population label and the others quantitative variables.

○ ci: if $c i=1$, the confidence intervals are added to $P_{S T}$ values. Default value: $c i=0$.

o csh: the $\frac{c}{h^{2}}$ value. Default value: $c s h=1$.

$\circ$ va: a vector containing the names or column numbers of the quantitative measures under consideration. If va $=0$, all the variables are selected. Default value: va $=0$.

o boot: the number of data frames generated to determine the confidence interval with the bootstrap method. Default value: boot $=1000$.

- Pw: the names of the two populations considered to obtain pairwise $P_{S T}$. Default value: $\mathrm{Pw}=0$, no pairwise analysis.

○ Rp: the names of the populations to be deleted. Default value: $\mathrm{Rp}=0$, no populations removed.

- Ri: the line numbers of individuals to be deleted. Default value: $\mathrm{Ri}=0$, no individuals removed.

- pe: the confidence level of the calculated interval. Default value: pe=0.95.

Let us apply the Pst function to the test dataset. The output from Pst will be a data frame:

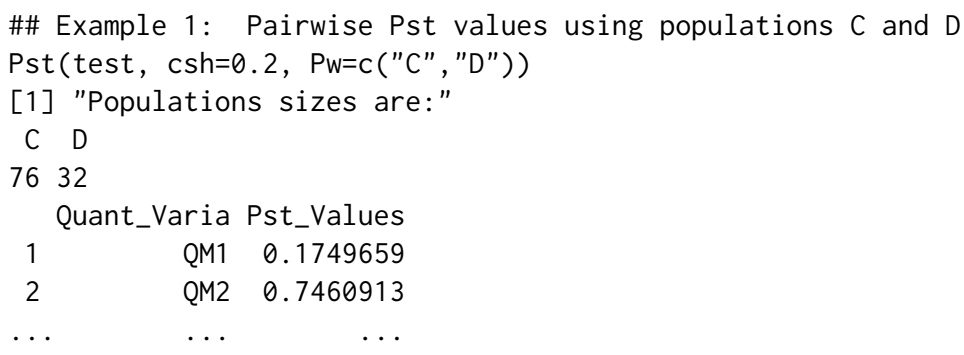


\#\# Example 2: Pst for the 2nd variable and QM7 with 99\% confidence intervals Pst (test, va=c(2, "QM7"), $c i=1$, boot=10000, $\mathrm{Ri}=c(5,117: 121)$, pe=0.99)

[1] "Populations sizes are:"

A B C D $\quad E$

$12767230 \quad 4$

Quant_Varia Pst_Values 99 \%_LowBoundCI 99 \%_UpBoundCI

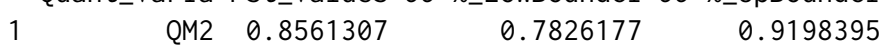

$2 \quad$ QM7 $0.8851413 \quad 0.7722856 \quad 0.9376501$

\section{Distribution of $P_{S T}$}

The bootstrapped $P_{S T}$ values output from BootPst form a distribution for the selected quantitative trait. In addition to arguments that are shared with Pst, the BootPst function has the following additional arguments specific to the bootstrap procedure:

- opt: if opt=0, all the boot values of $P_{S T}$ are returned; if opt="ci", the ordered values and the confidence interval are returned; and if opt="hist", the ordered values and the distribution histogram of $P_{S T}$ are returned. Default value: opt $=0$.

$\circ$ va: the name or column number of the quantitative measure considered.

- bars: the maximum number of bars the histogram may have. On the $x$-axis, the interval $[0,1]$ is divided into bars parts (there may exist unfilled bars). Default value: bars $=20$.

The output from the BootPst function is a vector with the bootstrapped values.

Let us apply the BootPst function to test dataset:

\#\# Example 1: Bootstrapped 95\% confidence intervals for three populations (B, C, and D). \#\# Note that populations $A$ and $E$ are dropped

BootPst(test, opt="ci", va="Body_length", Rp=c("A", "E"))

[1] "The studied quantitative variable is:"

[1] "Body_length"

[1] "Populations sizes are:"

B C D

767632

[1] "95\% confidence interval determined by 1000 bootstrap values:"

[1] $0.8757057 \quad 0.9585423$

$\begin{array}{lllllllllll}{[1]} & 0.7938426 & 0.8338286 & 0.8510682 & 0.8512374 & 0.8545911 & 0.8551115 & 0.8552097\end{array}$

[8] $0.8637057 \quad 0.8641575 \quad 0.8644145 \quad 0.8659723 \quad 0.8671139 \quad 0.86712650 .8676122$

$\begin{array}{lllllllllll}{[15]} & 0.8686147 & 0.8702277 & 0.8708352 & 0.8711419 & 0.8718030 & 0.8721783 & 0.8734932\end{array}$

...

$\left[\begin{array}{llllllll}995 & 0.9621794 & 0.9625852 & 0.9634700 & 0.9644283 & 0.9650500 & 0.9689611\end{array}\right.$

\#\# Example 2: Histogram for the trait in column 3 (output in Figure 1)

BootPst (test, opt="hist", va=3, bars=50)

[1] "The studied quantitative variable is:"

[1] "QM3"

[1] "Populations sizes are:"

A B C D

$127676 \quad 32 \quad 4$

[1] "1000 bootstrap values and Pst distribution:"

$\begin{array}{llllllllll}{[1]} & 0.1062747 & 0.1076470 & 0.1269888 & 0.1593121 & 0.1775196 & 0.2050347 & 0.2111617\end{array}$

[8] $0.2327508 \quad 0.2401064 \quad 0.2487401 \quad 0.25881790 .2589942 \quad 0.2623706 \quad 0.2722956$

$\begin{array}{lllllllll}{[15]} & 0.2827915 & 0.2860497 & 0.2935858 & 0.2947525 & 0.2954878 & 0.2995198 & 0.3003267\end{array}$

$\ldots$

$\left[\begin{array}{lllllllll}9955] & 0.8211326 & 0.8253874 & 0.8293417 & 0.8318546 & 0.8420100 & 0.8635299\end{array}\right.$

\section{Variations of $P_{S T}$ values and visual comparison with Wright's $F_{S T}$ index}

Brommer (2011) and Lima (2012) offer plots that demonstrate how $F_{S T}$ and $P_{S T}$ depend on the $\frac{c}{h^{2}}$ ratio. The Pstat package provides plotting tools to perform these analyses with the function TracePst. Arguments specific to TracePst include: 


\section{Pst distribution:}

QM3

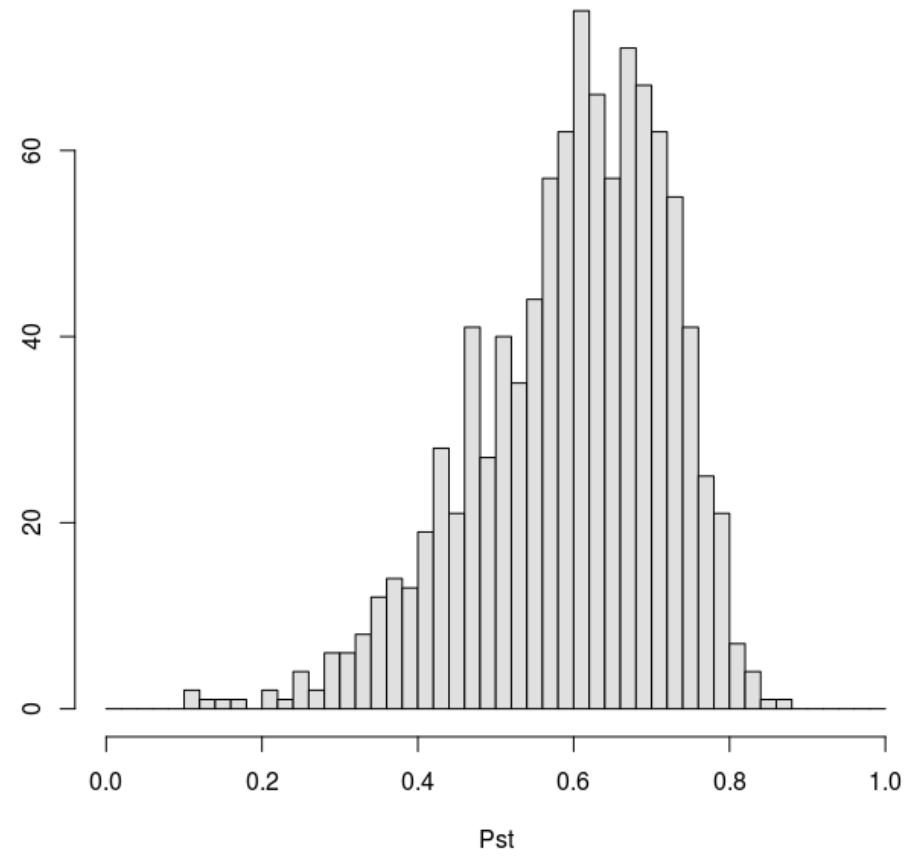

Figure 1: $P_{S T}$ distribution histogram of QM3.

- va: a vector containing the selected variables names or numbers (i.e. those of the quantitative measures considered). If $v a=0$, all the variables are selected. Default value: va $=0$.

$\circ$ ci: if $c i=1$, the confidence interval of $P_{S T}$ is plotted. Default value: $c i=1$.

Fst: the value of Wright's $F_{S T}$, if available. Default value: Fst=-1, value of $F_{S T}$ is unavailable.

o xm: $x$-axis maximum. Default value: $x m=2$.

o pts: the number of points used to plot the curves. Default value: $p t s=30$.

Let us apply the TracePst function to the test dataset. The plots output are in Figure 2.

\# Aitchison adjustment method:

trans_test=AitTrans(test)

\# Plots illustrating how comparisons between Fst and Pst depends on $\mathrm{c} / \mathrm{h}^{\wedge} 2$ :

TracePst(trans_test, Fst $=0.3, \mathrm{xm}=3$ )

[1] "Populations sizes are:"

A B C D E

$\begin{array}{lllll}12 & 76 & 76 & 32 & 4\end{array}$

\section{Conclusion}

The use of $P_{S T}$ versus $F_{S T}$ comparison has increased rapidly in the last few years in the field of evolutionary and ecological genetics. The Pstat package is the counterpart of existing $\mathrm{R}$ packages dealing with $F$-statistics. It calculates $P_{S T}$ values, and also provides bootstrapped confidence intervals, several graphical tools, as well as three ways of transforming data to remove variation resulting from allometric growth.

\section{Bibliography}

J. Aitchison. The Statistical Analysis of Compositional Data. Chapman and Hall, London, New York, 1986. 416 pp. [p448, 449] 

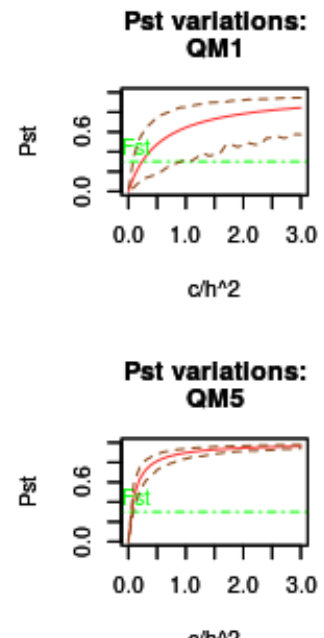

$c / h^{\wedge} 2$

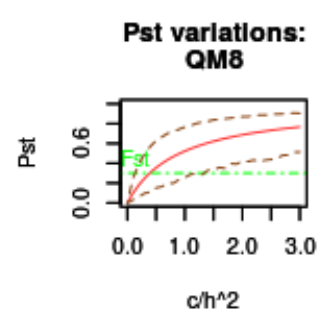

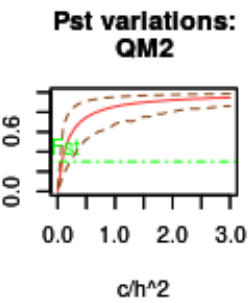

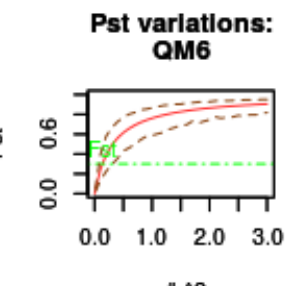

$c / h^{\wedge} 2$

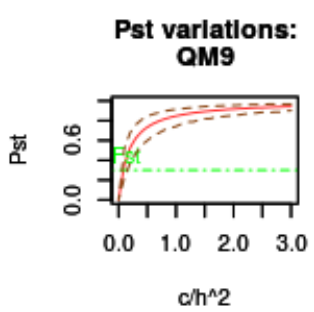

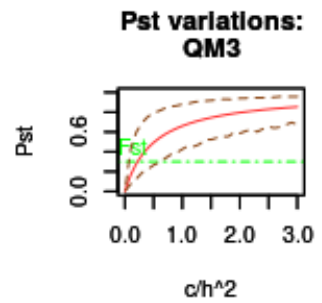
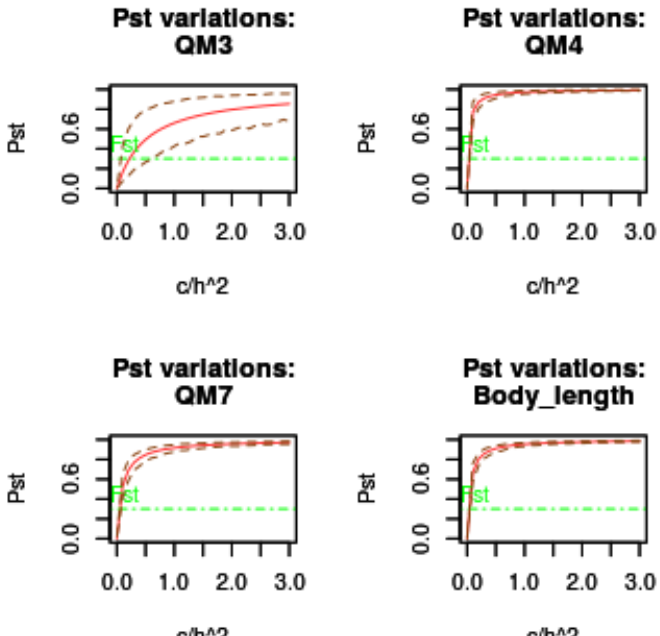

Pst varlations: Body_length

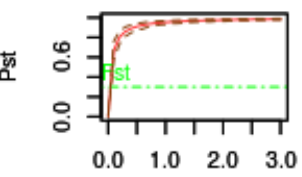

$c / h^{\wedge} 2$

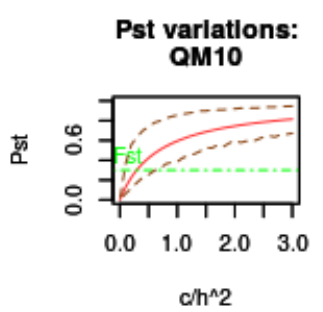

Figure 2: Plots illustrating comparisons between $F_{S T}$ and $P_{S T}$. The horizontal dotted green line marks the value of $F_{S T} . P_{S T}$ values and associated $95 \%$ confidence intervals are plotted in red.

J. E. Brommer. Whither Pst? The approximation of Qst by Pst in evolutionary and conservation biology. Journal of Evolutionary Biology, 24:1160-1168, 2011. URL https://doi . org/10.1111/j .14209101.2011.02268.x. [p447, 451]

B. Efron and R. Tibshirani. An Introduction to the Bootstrap. Chapman and Hall, New York, 1993. [p450]

J. Goudet. HIERSTAT, a package for R to compute and test hierarchical F-statistics. Molecular Ecology Resources, 5(1):184-186, 2005. URL https://doi.org/10.1111/j.1471-8286.2004.00828.x. [p448]

Y. He, R. Li, J. Wang, S. Blanchet, and S. Lek. Morphological variation among wild populations of chinese rare minnow (Gobiocypris rarus): Deciphering the role of evolutionary processes. Zoological Science, 30:475-483, 2013. URL https://doi.org/10.2108/zsj.30.475. [p447]

K. Keenan, P. McGinnity, T. F. Cross, W. W. Crozier, and P. A. Prodöhl. diveRsity: An R package for the estimation and exploration of population genetics parameters and their associated errors. Methods in Ecology and Evolution, 4(8):782-788, 2013. URL https://doi .org/10.1111/2041-210X.12067. [p447]

B. Kuhry and L. F. Marcus. Bivariate linear models in biometry. Systematic Zoology, 26(2):201-209, 1977. URL https://doi .org/10.2307/2412842. [p448]

T. Leinonen, J. M. Cano, H. Makinen, and J. Merilä. Contrasting patterns of body shape and neutral genetic divergence in marine and lake populations of threespine sticklebacks. Journal of Evolutionary Biology, 19:1803-1812, 2006. URL https://doi .org/10.1111/j.1420-9101.2006.01182.x. [p447]

T. Leinonen, R. J. McCairns, R. B. O'Hara, and J. Merilä. Qst-Fst comparisons: Evolutionary and ecological insights from genomic heterogeneity. Nature Reviews Genetics, 14:179-190, 2013. URL https://doi.org/10.1038/nrg3395. [p447]

M. R. Lima. Genetic and morphometric divergence of an invasive bird: The introduced house sparrow (Passer domesticus) in Brazil. PloS One, 7(12), 2012. URL https://doi .org/10.1371/journal . pone. 0053332. [p447, 451]

J. Merilä and P. Crnokrak. Comparison of genetic differentiation at marker loci and quantitative traits. Journal of Evolutionary Biology, 14:892-903, 2001. URL https://doi .org/10.1046/j.14209101.2001.00348.x. [p447] 
S. G. Michalski and W. Durka. Separation in flowering time contributes to the maintenance of sympatric cryptic plant lineages. Ecology and Evolution, 5(11):2172-2184, 2015. URL https://doi . org/10.1002/ece3.1481. [p448]

K. B. Mobley, D. Lussetti, F. Johansson, G. Englund, and F. Bokma. Morphological and genetic divergence in swedish postglacial stickleback (Pungitius pungitius) populations. BMC Evolutionary Biology, 2011. URL https://doi .org/10.1186/1471-2148-11-287. [p447]

J. D. Reist. An empirical evaluation of several univariate methods that adjust for size variation in morphometric data. Canadian Journal Zoology, 63:1429-1439, 1985. URL https://doi .org/10.1139/ z85-213. [p448, 449]

J. Reynolds, B. S. Weir, and C. C. Cockerham. Estimation of the coancestry coefficient: Basis for a short-term genetic distance. Genetics, 105:767-779, 1983. [p447]

C. Shinn, S. Blanchet, G. Loot, S. Lek, and G. Grenouillet. Phenotypic variation as an indicator of pesticide stress in gudgeon: Accounting for confounding factors in the wild. Science of the Total Environment, 538:733-752, 2015. URL https://doi .org/10.1016/j.scitotenv.2015.08.081. [p447]

K. Spitze. Population structure in Daphnia obtusa: Quantitative genetic and allozymic variation. Genetics, 135:367-374, 1993. [p447]

S. Wright. The genetical structure of populations. Annals of Human Genetics, 15:323-354, 1951. URL https://doi.org/10.1111/j.1469-1809.1949.tb02451.x. [p447]

Stéphane Blondeau Da Silva, corresponding author XLIM-Mathis, UMR $n^{\circ} 7252$ CNRS-Université de Limoges

123, avenue Albert Thomas 87060 Limoges

France

blondeaudasilva@xlim.fr

Anne Da Silva

INRA, UMR $n^{\circ} 1061$ Génétique Moléculaire Animale

Université de Limoges, UMR $n^{\circ} 1061$ Génétique Moléculaire Animale

123, avenue Albert Thomas 87060 Limoges

France

anne.blondeau@unilim. fr 